\title{
Ergogenic effects of caffeine and sodium bicarbonate supplementation on intermittent exercise performance preceded by intense arm cranking exercise
}

\author{
Matthaus Marriott ${ }^{1}$, Peter Krustrup ${ }^{1,2}$ and Magni Mohr $r^{3,4^{*}}$
}

\begin{abstract}
Background: Caffeine and sodium bicarbonate ingestion have been suggested to improve high-intensity intermittent exercise, but it is unclear if these ergogenic substances affect performance under provoked metabolic acidification. To study the effects of caffeine and sodium bicarbonate on intense intermittent exercise performance and metabolic markers under exercise-induced acidification, intense arm-cranking exercise was performed prior to intense intermittent running after intake of placebo, caffeine and sodium bicarbonate.

Methods: Male team-sports athletes $(n=12)$ ingested sodium bicarbonate $\left(\mathrm{NaHCO}_{3} ; 0.4 \mathrm{~g} \cdot \mathrm{kg}^{-1}\right.$ b.w.), caffeine (CAF; $6 \mathrm{mg} \mathrm{kg}^{-1}$ b.w.) or placebo (PLA) on three different occasions. Thereafter, participants engaged in intense arm exercise prior to the Yo-Yo intermittent recovery test level-2 (Yo-Yo IR2). Heart rate, blood lactate and glucose as well as rating of perceived exertion (RPE) were determined during the protocol.

Results: $\mathrm{CAF}$ and $\mathrm{NaHCO}_{3}$ elicited a 14 and 23\% improvement $(P<0.05)$, respectively, in Yo-Yo IR2 performance, post arm exercise compared to PLA. The $\mathrm{NaHCO}_{3}$ trial displayed higher [blood lactate] $(P<0.05)$ compared to CAF and PLA (10.5 \pm 1.9 vs. $8.8 \pm 1.7$ and $7.7 \pm 2.0 \mathrm{mmol}^{-L^{-1}}$, respectively) after the Yo-Yo IR2. At exhaustion CAF demonstrated higher $(P<0.05)$ [blood glucose] compared to PLA and $\mathrm{NaHCO}_{3}(5.5 \pm 0.7$ vs. $4.2 \pm 0.9$ vs. $4.1 \pm$ 0.9 mmol.L ${ }^{-1}$, respectively). RPE was lower $(P<0.05)$ during the Yo-Yo IR2 test in the $\mathrm{NaHCO}_{3}$ trial in comparison to CAF and PLA, while no difference in heart rate was observed between trials.
\end{abstract}

Conclusions: Caffeine and sodium bicarbonate administration improved Yo-YO IR2 performance and lowered perceived exertion after intense arm cranking exercise, with greater overall effects of sodium bicarbonate intake.

Keywords: Yo-Yo IR2 test performance, Fatigue, Blood lactate, Rating of perceived exertion, Team sport athletes

\section{Introduction}

Fatigue during high-intensity intermittent exercise is complex and multifaceted. Early speculation regarding the aetiology of fatigue commends that high rate of lactic acid production and a concomitant fall in blood and muscle $\mathrm{pH}$ [1], which may have multiple indirect and direct impairing effects on centrally and peripherally mediated fatigue-resistance. Caffeine and sodium bicarbonate

\footnotetext{
* Correspondence: magnim@setur.fo

${ }^{3}$ Faculty of Natural and Health Sciences, University of the Faroe Islands, Jónas Broncks gøta 25. 3rd floor, Tórshavn, Faroe Islands

${ }^{4}$ Center of Health and Human Performance, Department of Food and

Nutrition, and Sport Science, University of Gothenburg, Gothenburg, Sweden Full list of author information is available at the end of the article
}

$\left(\mathrm{NaHCO}_{3}\right)$ are two supplements frequently consumed to elicit ergogenic effects on high-intensity exercise performance [2].

Effects of caffeine on intense intermittent exercise performance have for example been studied by Stuart et al. [3] demonstrating improved repeated sprint performance during a simulated rugby game trial. Moreover, team-sport athletes improved both total work and mean power output during an intermittent cycle sprint protocol [4] and Yo-Yo IR2 performance by $16 \%$ after caffeine intake [5]. However, Glaister et al. [6] showed that although the fastest sprint time in a repeated sprint test was observed with caffeine intake, the magnitude of 
fatigue in the caffeine condition appeared to be greater compared to a placebo trial. Furthermore, no ergogenic effect has been found on sprint performance during the Loughborough intermittent shuttle test [7]. Thus, the effects of caffeine intake on intense intermittent exercise protocols are equivocal.

$\mathrm{NaHCO}_{3}$ ingested 90-150 min prior exercise has been used as an ergogenic aid for athletic events highly dependent on anaerobic glycolysis, since the ergogenic potential that $\mathrm{NaHCO}_{3}$ might elicit is suggested to depend upon the demands of the activity being sufficient to induce performance inhibiting levels of metabolic acidosis [8]. $\mathrm{NaHCO}_{3}$ ingestion has been reported to improve competitive and laboratory-based protocols lasting 1-7 min including swimming, middle distance running, rowing and repeated sprinting [9]. In addition, improvement in performance during a repeated sprint protocol is reported [10]. However, other studies are less affirmative and demonstrating no performance enhancing effects on high-intensity intermittent cycling [11]. Further discrepancies have been illustrated by Cameron et al. [12] whereby no benefits were observed during a highintensity rugby-specific training session followed by a repeated-sprint test. The absence of effects within the aforementioned studies is potentially due to insufficient metabolic taxation.

The Yo-Yo Intermittent Recovery test level 2 (Yo-Yo IR2) consists of 20-m shuttle runs at progressive running speed and has a high anaerobic energy turnover [13]. Thus, in order to examine the effect of caffeine and $\mathrm{NaHCO}_{3}$ intake on high-intensity intermittent exercise the Yo-Yo IR2 test can be utilized. Moreover, engaging in intense arm exercise prior to repeated high-intensity running elevates the levels of leg muscle and blood [lactate] and $\left[\mathrm{H}^{+}\right][14]$, as well as increasing the accumulation rate in muscle interstitial $\left[\mathrm{K}^{+}\right]$resulting in decreased knee extensor exercise performance [15]. Therefore, intense upper-body exercise prior to running exercise can be applied to induce pre-exercise muscle acidosis and high metabolic disturbance without exercising the legs.

Thus, the aim of the present study was therefore to compare the effects of caffeine and $\mathrm{NaHCO}_{3}$ supplementation 70-90 min prior to exercise, respectively, on Yo-Yo IR2 performance and physiological response to intense intermittent exercise with prior metabolic acidosis induced by intense arm cranking exercise.

\section{Methods}

\section{Participants}

Twelve healthy male participants involved in sub-elite team-sports (age: $20.8 \pm 1.4( \pm \mathrm{SD})$ yrs.; height: $183 \pm$ $7 \mathrm{~cm}$; body mass: $78.9 \pm 5.4 \mathrm{~kg}$ ) volunteered to participate in this study. Participants gave their written informed consent to participate prior to the experimental procedures and the study conforms the ethical guidelines of the Declaration of Helsinki. The study was approved by the University of Exeter Ethics Committee.

\section{Design}

The participants reported to the laboratory on five separate occasions with at least four days between visits. On the initial visit to the laboratory the participants were familiarised to the arm exercise protocol conducted on an upper body arm cranking ergometer (Lode BV, Angio, Netherlands) to determine individual specific power outputs as previously described [15]. On the second occasion participants performed a baseline, control (CON) Yo-Yo IR2 that they were familiarized to prior to the study [13]. The CON-trial was performed without any supplementation or prior arm-exercise. Participants were then assigned in a single-blind, randomized, crossover design to receive placebo (PLA; plain flour), caffeine (CAF) or sodium bicarbonate $\left(\mathrm{NaHCO}_{3}\right)$ supplementation.

\section{Experimental procedure}

On each experimental visit, participants were asked to report to the laboratory $100 \mathrm{~min}$ prior to the initiation of the Yo-Yo IR2 in a fully hydrated state and $\geq 2 \mathrm{~h}$ postprandial. The tests were carried out at the same time of the day $( \pm 1 \mathrm{~h})$. The participants were not permitted to consumed alcohol $24 \mathrm{~h}$ prior to testing or to take any other form of dietary supplements for the duration of the study and to avoid strenuous exercise $24 \mathrm{~h}$ preceding each experimental trial. In addition the participants were asked to avoid food items containing caffeine prior to the experimental days. Moreover, the food intake was noted the day prior to the first test trial and replicated prior to the remaining trials. Upon arrival to the laboratory participants were fitted with a heart rate (HR) monitor (Polar Electro, Kempele, Finland) and a baseline fingertip capillary blood sample was obtained. Participants were then given either CAF or PLA (blinded) or $\mathrm{NaHCO}_{3}$ to orally ingest under the supervision of the researchers. $70 \mathrm{~min}$ before the start of the Yo-Yo IR2 either CAF or PLA was taken orally in gelatine capsule (6 mg. $\mathrm{kg}^{-1}$ body mass; $474 \pm 31 \mathrm{mg}$; see Mohr et al. [5]). $\mathrm{NaHCO}_{3}$ was ingested orally in 21-25 gelatine capsules (0.4 g. $\mathrm{kg}^{-1}$ body mass; $\left.31.6 \pm 1.6 \mathrm{~g}\right)$ and supplementation began 90 min prior to the start of the Yo-Yo IR2 with $7 \mathrm{ml} . \mathrm{kg}^{-1}$ of water drank ad libitum over a $30 \mathrm{~min}$ period as described by Carr et al. [9]. The respective $\mathrm{NaHCO}_{3}$ intake protocol has in a pilot study been demonstrated to markedly raise the blood $\mathrm{HCO}_{3}^{-}$concentration (data not shown).

The arm exercise protocol lasted for $17 \mathrm{~min}$, during which the participants maintained a constant cadence $(80 \pm 5$ RPM $)$ at individualised power outputs (157.6 \pm 
$7.4 \mathrm{~W})$. The protocol, adapted from others $[14,15]$ consisting of $4 \times 1$-min and $1 \times 1.5$-min exercise periods separated by $0.5-\mathrm{min}$ recovery, followed by $4.5 \mathrm{~min}$ of recovery and a final 1-min exercise period. Immediately after completion of the arm exercise, a blood sample was taken. The Yo-Yo IR2 began $4 \mathrm{~min}$ after the completion of the arm exercise protocol corresponding to 70 min post CAF and PLA intake and 90 min after the $\mathrm{NaHCO}_{3}$ ingestion. The Yo-Yo IR2 test was completed on a wooden surface and consists of repeated two $20-\mathrm{m}$ runs at a progressively increased speed controlled by audio bleeps from a CD player [13]. The test-leader controlling the Yo-Yo IR2 test was blinded in relation to drug-treatment.

Fingertip capillary $300 \mu \mathrm{L}$ blood samples were collected in heparin-fluoride coated Microvette CB 300 tubes (Sarstedt Ltd, UK) which were immediately stored on ice and subsequently analysed to determine blood [lactate] and [glucose] after the protocol [5] using a YSI 2500 Lactate Analyser (YSI, Yellow Springs, US) having a test-retest coefficient of variance of $<2 \%$. Blood samples were obtained: prior to supplementation on arrival to the laboratory, immediately after arm exercise, immediately after Yo-Yo IR2 exhaustion, 1, 3 and $5 \mathrm{~min}$ post exhaustion. Heart rate was determined during the entire protocol while rating of perceived exertion (RPE) values using the Borg scale [16] were recorded during the YoYo IR2 at 160, 280, 440, $600 \mathrm{~m}$ and at exhaustion.

\section{Statistical analyses}

Differences between baseline Yo-Yo IR2 performance and PLA Yo-Yo IR2 after arm exercise were analysed using a paired-samples $t$-test. A one-way repeated measures ANOVA was used to determine the influence of supplementation on the performance in the Yo-Yo IR2 after intense arm exercise. Where analyses revealed a significant main effect, the origin of this effect was determined by Bonferroni adjusted post hoc paired $t$-tests. Differences in plasma [glucose], [lactate], HR and RPE between the three conditions (PLA, CAF and $\mathrm{NaHCO}_{3}$ ) were analysed using multiple separate two-way repeated measures ANOVAs (supplement $\mathrm{x}$ time). Significant main effects were further analysed by Bonferroni adjusted post hoc paired $t$-tests. All repeated measures data was checked for the assumption of sphericity using Mauchly's test. All data were analysed using the statistical software package SPSS (version 20). Statistical significance was accepted at $P<0.05$. Results are presented as means $\pm \mathrm{SD}$.

\section{Results}

\section{Performance}

Yo-Yo IR2 performance was reduced $(\mathrm{P}<0.05)$ by $41 \%$ when the test was preceded by an intense intermittent arm cranking (CON: $696 \pm 185$ m vs. PLA: $413 \pm 121 \mathrm{~m}$; $P<0.01$, Figure 1A). However, after $\mathrm{CAF}$ and $\mathrm{NaHCO}_{3}$ supplementation Yo-Yo IR2 performance was 14 and $23 \%$ higher $(\mathrm{P}<0.05)$ compared to PLA $(480 \pm 113$ and $540 \pm 138 \mathrm{~m}$, respectively), with a greater $(\mathrm{P}<0.05) \mathrm{im}$ provement in $\mathrm{NaHCO}_{3}$ than CAF (Figure 1B).

\section{Blood metabolites}

Baseline blood [Lactate] was similar $(0.9 \pm 0.3,1.0 \pm 0.5$ and $\left.0.9 \pm 0.6 \mathrm{mmol} . \mathrm{L}^{-1} ; \mathrm{P}>0.05\right)$ for PLA, CAF and $\mathrm{NaHCO}_{3}$, respectively, but increased $(P<0.01)$ post arm crank $\left(7.3 \pm 1.8,7.4 \pm 1.5\right.$ and $8.3 \pm 1.8 \mathrm{mmol}^{-1} \mathrm{~L}^{-1}$, respectively, Figure $2 \mathrm{~A}$ ) with no significant differences between the three trials, although there was a trend $(P=0.09)$ for higher blood [lactate] for the $\mathrm{NaHCO}_{3}$ trial. Blood [lactate] did not change $(P>0.05)$ during Yo-Yo IR2 for PLA at exhaustion $\left(7.7 \pm 2.0 \mathrm{mmol} . \mathrm{L}^{-1}\right)$, but rose $(P<0.05)$ for both $\mathrm{CAF}$ and $\mathrm{NaHCO}_{3}\left(8.8 \pm 1.7\right.$ vs. $10.5 \pm 1.9 \mathrm{mmol} . \mathrm{L}^{-1}$, respectively, Figure 2A). Between group comparisons revealed that blood [lactate] values were similar between PLA and CAF, but higher $(P<0.01)$ for the $\mathrm{NaHCO}_{3}$ trial compared to PLA and CAF trials at exhaustion (Figure 2A). At 1 min post exhaustion PLA blood [lactate] rose (9.4 \pm $\left.1.8 \mathrm{mmol. \textrm {L } ^ { - 1 }} ; P<0.05\right)$ compared to exhaustion whereas $\mathrm{CAF}$ and $\mathrm{NaHCO} 3$ remained unchanged $(P>0.05)$ and blood [lactate] in the $\mathrm{NaCHO}_{3}$ trial still remained higher $\left(11.6 \pm 1.7\right.$ mmol.L $\left.\mathrm{L}^{-1} ; P<0.01\right)$ when compared to PLA and CAF. At 3 min post Yo-Yo IR2 [lactate] was not different between groups $\left(8.1 \pm 1.9,8.5 \pm 2.2\right.$ and $8.8 \pm 1.7 \mathrm{mmol} . \mathrm{L}^{-1}$; $P>0.05)$ PLA, CAF and $\mathrm{NaHCO}_{3}$, respectively. At $5 \mathrm{~min}$ into recovery Yo-Yo IR2 blood [lactate] was reduced in the PLA trial when compared to CAF and $\mathrm{NaHCO}_{3}(7.2 \pm 2.2$ vs. $8.5 \pm 2.3$ and $9.1 \pm 1.9$ mmol. $\mathrm{L}^{-1} ; P<0.05$, respectively) whereas CAF and $\mathrm{NaCHO}_{3}$ were similar at this time point $(P>0.05)$.

Baseline blood [glucose] was not significantly different between trials $(4.5 \pm 0.5$ vs. $4.2 \pm 0.4$ vs. $4.4 \pm 0.9 \mathrm{mmol}$. $\mathrm{L}^{-1} ; P>0.05$, Figure 2B) for PLA, CAF and $\mathrm{NaHCO}_{3}$, respectively. For all trials at Yo-Yo IR2 exhaustion there was no difference $(P>0.05)$ in blood [glucose] within and between the supplements $(3.9 \pm 0.9$ vs. $4.5 \pm 1.1$ vs. $4.2 \pm 0.8 \mathrm{mmol} . \mathrm{L}^{-1}$, respectively, Figure $2 \mathrm{~B}$ ). In the PLA and $\mathrm{NaHCO}_{3}$ trials blood [glucose] rose $(\mathrm{P}<0.05)$ between exhaustion and $1 \mathrm{~min}$ post Yo-Yo IR2 $(4.9 \pm 0.9$ and $4.9 \pm 0.7 \mathrm{mmol} \cdot \mathrm{L}^{-1}$ ). In the CAF trial blood [glucose] rose by $30 \%$ at the end of the protocol (Figure 2B), compared to the PLA $(P<0.05)$. However, no differences $(P>0.05)$ were in [glucose] between PLA and $\mathrm{NaHCO}_{3}$ at any protocol time points (Figure 2B). Between group comparisons revealed that at $5 \mathrm{~min}$ post Yo-Yo IR2 blood [glucose] in the CAF trial was greater $(P<0.05)$ compared to the PLA and $\mathrm{NaHCO}_{3}(5.5 \pm 0.7$ vs. $4.2 \pm 0.9$ and $4.1 \pm 0.9 \mathrm{mmol} . \mathrm{L}^{-1}$, respectively). 


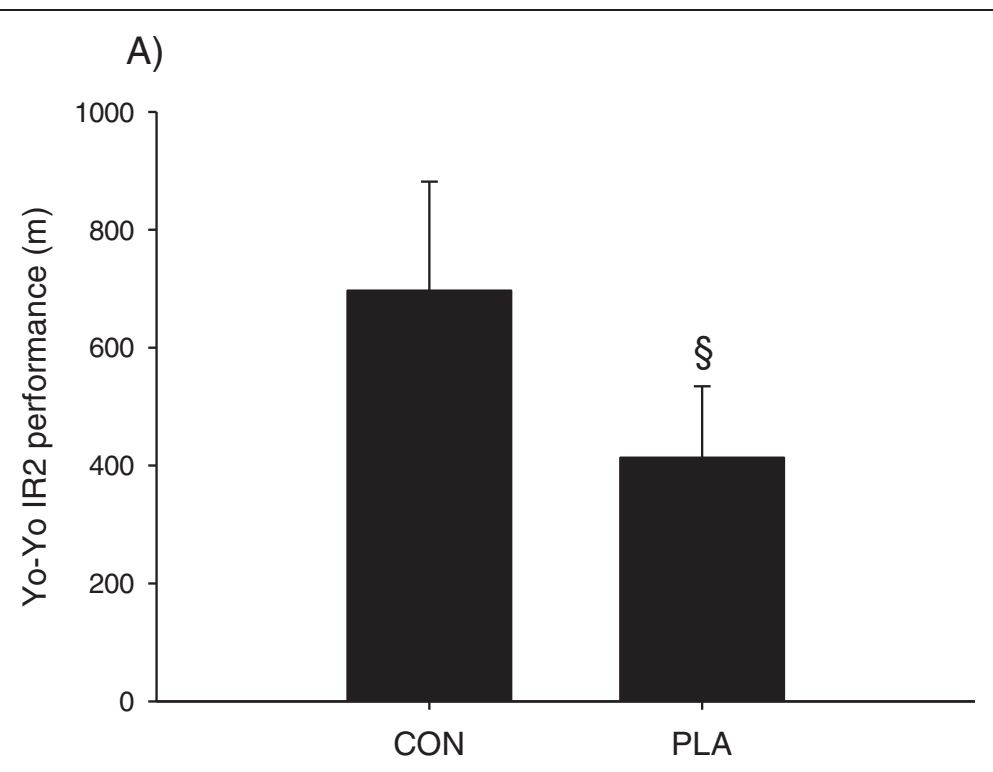

B)

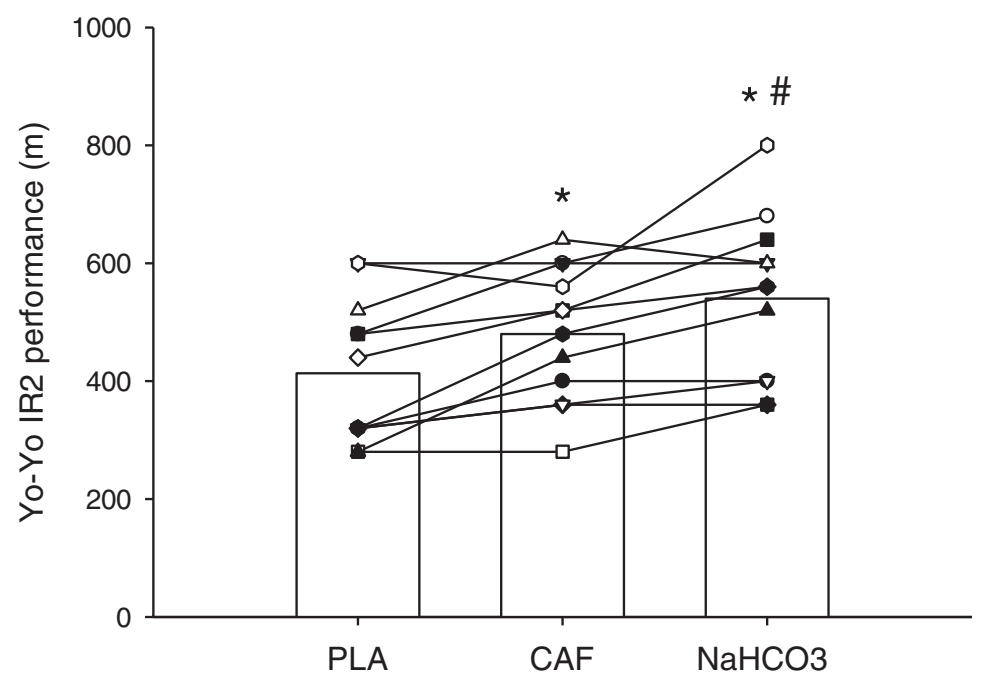

Figure 1 Yo-Yo Intermittent Recovery level 2 test (Yo-Yo IR2) performance in control (CON) and placebo (PLA) trials (A) and individual Yo-Yo IR2 performance during PLA and after caffeine (CAF) and sodium bicarbonate $\left(\mathrm{NaHCO}_{3}\right)$ supplementation $(B)(n=12)$. §: Denotes a significant difference from CON. *Denotes a significant difference from PLA. \# Denotes a significant difference from CAF. Significance level $\mathrm{P}<0.05$

\section{Rating of perceived exertion and heart rate loading}

Between group comparisons revealed that RPE was reduced $(P<0.05)$ in the $\mathrm{NaHCO}_{3}$ trials at both $160 \mathrm{~m}$ and $280 \mathrm{~m}(160 \mathrm{~m}: 15.3 \pm 2.3$ and $15.0 \pm 1.6$ vs. $13.1 \pm$ 2.0), (280 m: $17.8 \pm 1.2$ and $17.3 \pm 1.8$ vs. $15.8 \pm 1.5)$ for PLA, CAF and $\mathrm{NaHCO}_{3}$, respectively (Figure 3). No differences $(P>0.05)$ were observed at Yo-Yo IR2 exhaustion $(19.3 \pm 1.0,19.3 \pm 0.9$ and $19.3 \pm 0.7$ in PLA, CAF and $\mathrm{NaHCO}_{3}$, respectively, Figure 3). Arm exercise caused a rise $(P<0.01)$ in heart rate during all trials $\left(179 \pm 7,178 \pm 8\right.$ and $177 \pm 9$ bts.min $\left.{ }^{-1}\right)$ for PLA, CAF and $\mathrm{NaHCO}_{3}$, respectively. At Yo-Yo IR2 exhaustion a further rise $(P<0.05)$ in heart rate for $\mathrm{CAF}$ and $\mathrm{NaHCO}_{3}$ was observed $\left(187 \pm 7\right.$ and $186 \pm 7$ bts. $\mathrm{min}^{-1}$, respectively). Comparisons between trials revealed that there was no difference between PLA, CAF and $\mathrm{NaHCO}_{3}$ at any time points during the testing protocol.

\section{Discussion}

The principle findings of the present study were that Yo-Yo IR2 performance after intense arm cranking exercise was markedly reduced compared to a control trial, while both caffeine and $\mathrm{NaHCO}_{3}$ intake improved fatigue resistance during the Yo-Yo IR2 test. $\mathrm{NaHCO}_{3}$ 


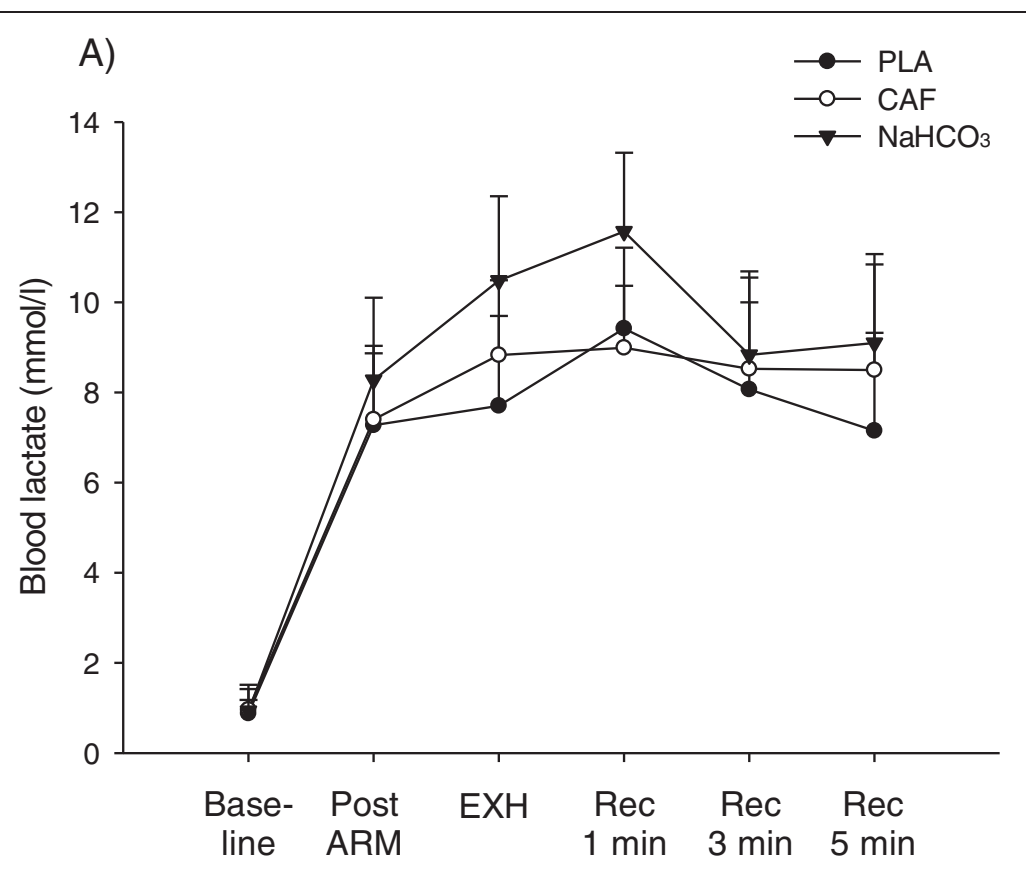

\section{B)}

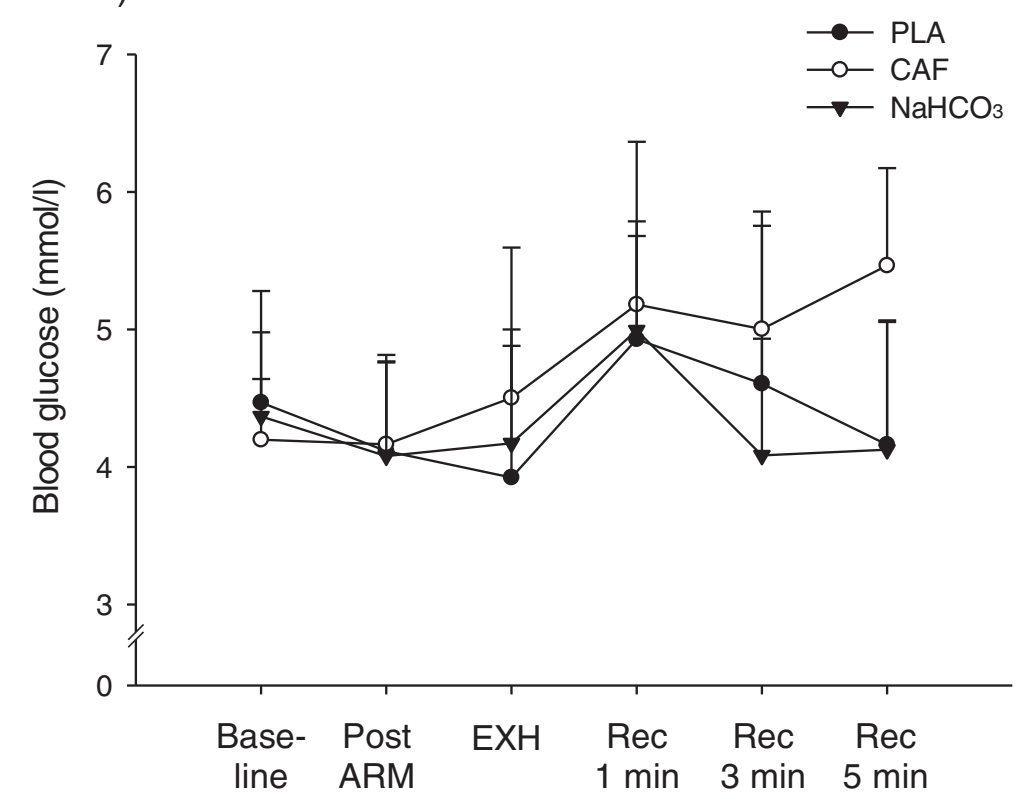

Figure 2 Capillary blood lactate (A) and glucose (B) concentrations before, during and after the Yo-Yo IR2 test protocol $(n=12)$ in the placebo (PLA), caffeine (CAF) and sodium bicarbonate $\left(\mathrm{NaHCO}_{3}\right)$ trials.

intake elicited a further ergogenic effect on Yo-Yo IR2 performance when performed after intense arm cranking exercise compared to that of caffeine. The present study is the first to report performance effects of caffeine and $\mathrm{NaHCO}_{3}$ intake using high-intensity intermittent running exercise preceded by intense arm cranking exercise and to demonstrate additional performance and perceptual benefits of $\mathrm{NaHCO}_{3}$ beyond that of caffeine.
The reduction in high-intensity running performance when performed after intense arm cranking exercise is consistent with previous research studying isolated muscle performance $[14,15]$. In the present study, Yo-Yo IR 2 performance was reduced by $41 \%$ when preceded by arm exercise, which is of a similar magnitude as the performance decrements of $35 \%$ and $\sim 48 \%$, respectively, observed during intense exhaustive one-legged knee extensor exercise performed after intense arm cranking 


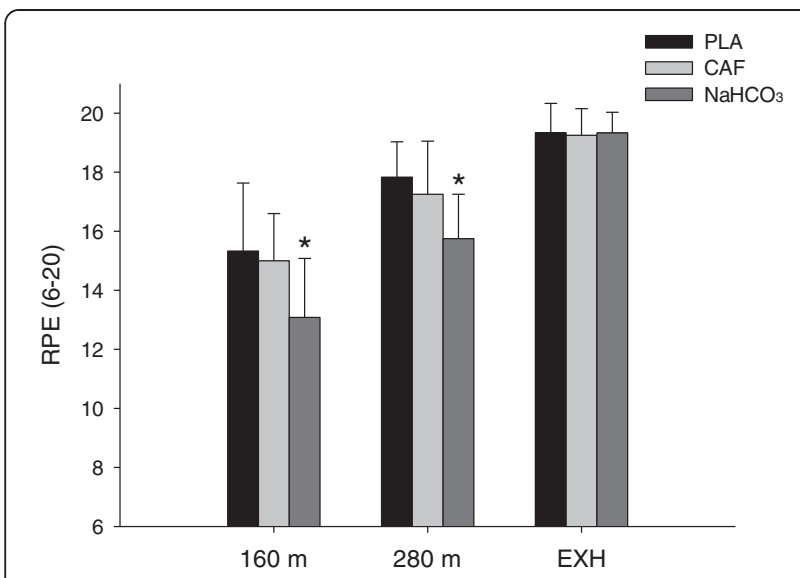

Figure 3 Rating of Perceived Exertion (RPE) at 160 and $280 \mathrm{~m}$ during the Yo-Yo IR2 test, as well as at exhaustion in PLA, CAF and $\mathrm{NaHCO}_{3}$. ${ }^{*}$ Denotes a significant difference from PLA. Significance level $\mathrm{P}<0.05$.

$[14,15]$. These two studies also showed that the arm exercise resulted in metabolic acidosis with arterial blood lactate being elevated by $\sim 11$-fold resting levels, as well as higher leg muscle lactate and $\mathrm{H}^{+}$concentrations [14] and elevated accumulation rate of leg muscle interstitial $\left[\mathrm{K}^{+}\right][15]$. In the present study capillary blood lactate was elevated $\sim 8$-fold after the arm cranking protocol, confirming that the metabolic environment was markedly altered prior to the Yo-Yo IR2 test compared to baseline conditions.

Yo-Yo IR2 performance was improved by $23 \%$ after $\mathrm{NaHCO}_{3}$ supplementation, which is supported by others $[9,10]$. Indeed performance enhancement in the final sprints of a repeated sprint test after $\mathrm{NaHCO}_{3}$ intake has been reported [10], indicating an ergogenic effect of $\mathrm{NaHCO}_{3}$ on fatigue resistance during high intensity exercise conditions performed under metabolic stress. However, some studies applying intense exercise protocols report no beneficial effect of $\mathrm{NaHCO}_{3}$ supplementation (for review see Carr et al. [9]). In the present study intense arm cranking markedly elevated blood lactate, confirming findings of an increased leg muscle lactate and $\mathrm{H}^{+}$concentration following the same arm protocol [14]. Part of the longer exercise time level in the $\mathrm{NaHCO}_{3}$-trial may be explained by an elevated $\mathrm{NaHCO}_{3}$ induced buffer capacity in the blood, which will increase the muscle-to-blood $\mathrm{H}^{+}$and lactate gradient. Since the monocarboxylate transporters (MCT) are gradient dependent [17], this will increase the removal of $\mathrm{H}^{+}$and lactate ions from the leg muscles before and during the Yo-Yo IR2 test. This may reduce the degree of intramuscular acidification and corresponding fatigue development [1]. The higher blood lactate levels were observed in the $\mathrm{NaHCO}_{3}$-trial, may also partly be a direct consequence of the greater fatigue resistance, and thereby higher glycolytic contribution and concomitant muscle lactate production compared to the caffeine and placebo trials. A limitation with the present study is that blood was not drawn during the Yo-Yo IR2 test, which have been done on other comparable studies $[5,13]$, so we are unable to compare the three intervention trial at fixed time points due to the different exercise times.

The intake of $\mathrm{NaHCO}_{3}$ may also affect the activity of sarcolemmal ion transporters. For example elevated extracellular $\mathrm{Na}^{+}$concentration may stimulate the $\mathrm{Na}^{+} / \mathrm{H}^{+}$ exchangers [18] and thereby increase the efflux of hydrogen ions from the exercising muscles and attenuate intramuscular acidification. In a study by Street et al. [19] muscle interstitial $\mathrm{pH}$ decreased gradually during graded exercise comparable to the Yo-Yo IR2 test. However, in a follow-up study sodium citrate was ingested prior to intense exercise, which on one hand elevated plasma $\mathrm{HCO}_{3}^{-}$and lowered the accumulation of muscle interstitial $\mathrm{H}^{+}$[18]. Finally, the accumulation rate of interstitial $\mathrm{K}^{+}$was significantly reduced, which was suggested to relate to less opening probability of the $K_{\text {ATP }}$ channels, which tend to open during intracellular acidification [20]. In addition, the elevated systemic $\mathrm{Na}^{+}$may directly stimulate the activity in the $\mathrm{Na}^{+}-\mathrm{K}^{+}$ATPase $[1,18]$. Thus, the intake of $\mathrm{NaHCO}_{3}$ may have elevated fatigue resistance by inducing maintenance of a more optimum intracellular $\mathrm{pH}[21]$ and/ or by enhancing $\mathrm{Na}^{+} / \mathrm{K}^{+}$pump activity and potentially $\mathrm{Na}^{+} / \mathrm{K}^{+} / 2 \mathrm{Cl}^{-}$co-transporter activity, contributing to lower muscle interstitial $\left[\mathrm{K}^{+}\right]$preserving sarcolemma excitability, allowing enhanced high intensity repeated muscular performance [1].

RPE was lower in $\mathrm{NaHCO}_{3}$-trial during the Yo-Yo IR2 test, which may suggest that centrally mediated mechanisms were affected. The participants experienced less exertion during the Yo-Yo IR2 in the $\mathrm{NaHCO}_{3}$-trial, and that although distance covered before exhaustion was increased the RPE values remained the same as the caffeine and placebo trials at the point of fatigue. Thus, a greater distance could be covered yet reporting an equal level of perceived fatigue at exhaustion. Peripheral changes may cause modulation of neural strategies via group III and IV muscle afferents which are widely distributed through the muscle and are responsive to chemical stimuli such as altered $\mathrm{H}^{+}$and $\mathrm{K}^{+}$[22]. Different mechanisms have been proposed by which such peripheral inputs might lead to modulation of motor neuron firing rates and muscle function including facilitation or inhibition of spinal reflexes, pre-synaptic inhibition of the group Ia afferents thus reducing inhibitory input into the motoneurons, and altered supraspinal drive [22]. Thus, part of the improved performance after $\mathrm{NaHCO}_{3}$ treatment may relate to less negative feedback from the muscle and thereby less 
effect on the descending drive to the motoneurons $[22,23]$.

In the present study caffeine increased Yo-Yo IR2 performance by $14 \%$ compared to the placebo-trial, which is in line with other findings [3,4]. Caffeine has multiple physiological effects that may promote fatigue resistance during intense exercise, such as elevating the catecholamine levels [24] and reducing muscle interstitial $\mathrm{K}^{+}$accumulation [5]. Peak blood glucose concentrations were higher in the caffeine-trial than placebo, which is in accordance with findings by others [5], indicating an elevated catecholamine response, which may facilitate the $\mathrm{Na}^{+}-\mathrm{K}^{+}$ATPase activity [25]. The arm protocol has been shown to increase the accumulation of interstitial potassium during leg exercise [15], and caffeine may reduce this effect by increasing the pump activity in the $\mathrm{Na}^{+}-\mathrm{K}^{+}$ APTase directly or via an elevated catecholamine response $[5,25]$. Finally caffeine may have an effect on central mechanisms associated with fatigue $[23,26]$, however, the RPE rating were not different between the caffeine and placebo trials in the present study. Caffeine is well-known to affect the central nervous system response mediated via antagonism of adenosine receptors, which dampens pain perception and attenuates fatigue [27]. However, during high-intensity intermittent exercise caffeine-induced effects on RPE appear to be negated [6], which is supported by this study.

Intriguingly, there were no differences in HR between the trials, suggesting that the aerobic demands were similar, yet participant's perception of fatigue was attenuated during the $\mathrm{NaHCO}_{3}$-trial, indicating that $\mathrm{NaHCO}_{3}$ in contrast to caffeine might impact upon central fatigue as well as peripheral fatigue.

The possibility of a 'high-responders' and 'low-responders' phenomena for both caffeine and $\mathrm{NaHCO}_{3}$ has been previously reported [27-29]. This effect was apparent in the present study, whereby three subjects and one subject, respectively, after caffeine and $\mathrm{NaHCO}_{3}$ intake, showed no improvement compared to PLA. Moreover five of the subjects reported experiencing some form of gastrointestinal discomfort after sodium bicarbonate intake; however this was apparently not detrimental to exercise performance.

\section{Conclusions}

The present study demonstrates that high-intensity intermittent exercise performance is impaired when preceded by intense arm exercise. However, fatigue resistance can be markedly improved by caffeine and $\mathrm{NaHCO}_{3}$ administration $70-90$ min prior to exercise. Moreover, for the first time $\mathrm{NaHCO}_{3}$ has been shown to elicit an improvement above that of caffeine in a sportspecific high-intensity intermittent test with concomitant reductions in RPE and higher blood lactate levels.
This study suggests that caffeine and $\mathrm{NaHCO}_{3}$ might be effective ergogenic aids for intermittent high-intensity exercise performance in sub-elite team sport athletes.

\section{Competing interests}

The authors declare that they have no competing interests.

\section{Authors' contributions}

MM and MM participated in the design of the study, and carried out the data collection in cooperation with PK. MM, PK and MM performed the data treatment including statistical analyses. MM and MM drafted the manuscript with help from PK. All authors read and approved the manuscript.

\section{Acknowledgement}

The authors would like to thank the athletes for committed participation. Moreover, we thank Dr. Nikolai Nordsborg, Dr. Sarah R. Jackman and Giorgios Ermidis for excellent technical assistance. The authors have no conflict of interest in relation to the study.

\section{Author details}

${ }^{1}$ Sport and Health Sciences, College of Life and Environmental Sciences, St. Luke's Campus, University of Exeter, Exeter, UK. ²Department of Nutrition, Exercise and Sports, Section of Human Physiology, Copenhagen Centre for Team Sport and Health, University of Copenhagen, Copenhagen, Denmark. ${ }^{3}$ Faculty of Natural and Health Sciences, University of the Faroe Islands, Jónas Broncks gøta 25. 3rd floor, Tórshavn, Faroe Islands. ${ }^{4}$ Center of Health and Human Performance, Department of Food and Nutrition, and Sport Science, University of Gothenburg, Gothenburg, Sweden.

Received: 15 December 2014 Accepted: 17 February 2015

Published online: 27 February 2015

\section{References}

1. Fitts R. Cellular mechanism of muscle fatigue. Physiol Rev. 1994;74:49-94

2. Kilding AE, Overton C, Gleave J. Effects of caffeine, sodium bicarbonate, and their combined ingestion on high-intensity cycling performance. Int J Sport Nutr Exerc Metab. 2012;22:175-83.

3. Stuart GR, Hopkins WG, Cook C, Cairns SP. Multiple effects of caffeine on simulated high-intensity team-sport performance. Med Sci Sports Exerc. 2005;37:1998-2005.

4. Schneiker KT, Bishop D, Dawson B, Hackett LP. Effects of caffeine on prolonged intermittent-sprint ability in team-sport athletes. Med Sci Sports Exerc. 2006;38:578-85.

5. Mohr M, Nielsen JJ, Bangsbo J. Caffeine intake improves intense intermittent exercise performance and reduces muscle interstitial potassium accumulation. J Appl Physiol. 2011;111:1372-9.

6. Glaister M, Howatson G, Abraham CS, Lockey RA, Goodwin JE, Foley P, et al. Caffeine supplementation and multiple sprint running performance. Med Sci Sports Exerc. 2008;40:1835-40.

7. Foskett A, Ali A, Gant N. Caffeine enhances cognitive function and skill performance during simulated soccer activity. Int J Sport Nutr Exerc Metab. 2009;19:410-23.

8. McNaughton LR, Siegler J, Midgley A. Ergogenic effects of sodium bicarbonate. Curr Sports Med Rep. 2008;7:230-6.

9. Carr AJ, Slater GJ, Gore CJ, Dawson B, Burke LM. Effect of sodium bicarbonate on $\left[\mathrm{HCO}_{3}^{-}\right]$, pH, and gastrointestinal symptoms. Int J Sport Nutr Exerc Metab. 2011;21:189-94.

10. Bishop D, Edge J, Davis C, Goodman C. Induced metabolic alkalosis affects muscle metabolism and repeated-sprint ability. Med Sci Sports Exerc. 2004;36:807-13.

11. Bishop D, Claudis B. Effects of induced metabolic alkalosis on prolonged intermittent-sprint performance. Med Sci Sports Exerc. 2005;37:759-67.

12. Cameron SL, MCLay-Cooke RT, Brown RC, Gray AR, Fairbairn KA. Increased blood $\mathrm{pH}$ but not performance with sodium bicarbonate supplementation in elite rugby union players. Int J Sport Nutr Exerc Metab. 2010;20:307-21.

13. Krustrup P, Mohr M, Nybo L, Nielsen JJ, Jensen JM, Bangsbo J. The Yo-Yo IR2 test: physiological response, reliability, and application to elite soccer. Med Sci Sports Exerc. 2006:38:1666-73.

14. Bangsbo J, Madsen K, Kiens B, Richter EA. Effect of muscle acidity on muscle metabolism and fatigue during intense exercise in man. J Physiol. 1996;495:587-96 
15. Nordsborg N, Mohr M, Pedersen LD, Nielsen JJ, Langberg H, Bangsbo J. Muscle interstitial potassium kinetics during intense exhaustive exercise: effect of previous arm exercise. Am J Physiol Regul Integr Comp Physiol. 2003;285:143-8.

16. Borg G. Borg's perceived exertion and pain scales. IL, USA: Champaign; 1998.

17. Juel C, Klarskov C, Nielsen JJ, Krustrup P, Mohr M, Bangsbo J. Effect of high-intensity intermittent training on lactate and $\mathrm{H}+$ release from human skeletal muscle. Am J Physiol Endocrinol Metab. 2004;286:e245-51.

18. Street D, Nielsen JJ, Bangsbo J, Juel C. Metabolic alkalosis reduces exercise-induced acidosis and potassium accumulation in human skeletal muscle interstitium. J Physiol. 2005;566:481-9.

19. Street D, Bangsbo J, Juel C. Interstitial pH in human skeletal muscle during and after dynamic graded exercise. J Physiol. 2001;537:993-8.

20. Davis NW, Standen NB, Stanfield PR. ATP-dependent potassium channels of muscle cells: their properties, regulation, and possible functions. J Bioenerg Biomembr. 1991;23:509-35.

21. Raymer GH, Marsh GD, Kowalchuk JM, Thompson RT. Metabolic effects of induced alkalosis during progressive forearm exercise to fatigue. J Appl Physiol. 2004;96:2050-6.

22. Gandevia SC. Spinal and supraspinal factors in human muscle fatigue. Physiol Rev. 2001;81:1725-89.

23. Nybo L, Secher NH. Cerebral perturbations provoked by prolonged exercise. Prog Neurobiol. 2004;72:223-61.

24. Greer F, McLean C, Graham TE. Caffeine, performance, and metabolism during repeated Wingate exercise tests. J Appl Physiol. 1998;85:1502-8.

25. Clausen $\mathrm{T}$. $\mathrm{Na}^{+}-\mathrm{K}^{+}$pump regulation and skeletal muscle contractility. Physiol Rev. 2003:83:1269-324.

26. Del Coso J, Muñoz-Fernández VE, Muñoz G, Fernández-Elías VE, Ortega JF, Hamouti $\mathrm{N}$, et al. Effects of a caffeine containing energy drink on simulated soccer performance. PLoS One. 2012;7:e31380.

27. Davis JK, Green JM. Caffeine and anaerobic performance: ergogenic value and mechanisms of action. Sports Med. 2009;39:813-32.

28. Wu CL, Shih MC, Yang CC, Huang MH, Chang CK. Sodium bicarbonate supplementation prevents skilled tennis performance decline after a simulated match. J Int Soc Sports Nutr. 2010;7:33-41.

29. Siegler JC, Marshall PW, Bray J, Towlson C, et al. Sodium bicarbonate supplementation and ingestion timing: does it matter? J Strength Cond Res. 2012;26:1953-8.

\section{Submit your next manuscript to BioMed Central and take full advantage of:}

- Convenient online submission

- Thorough peer review

- No space constraints or color figure charges

- Immediate publication on acceptance

- Inclusion in PubMed, CAS, Scopus and Google Scholar

- Research which is freely available for redistribution 